\title{
Stellungnahme der AG pneumologische Altersmedizin zu COVID-19 bei geriatrischen Patienten
}

\section{Recommendations for the Treatment of Elderly Patients with COVID-19 from the Taskforce for Gerontopneumology}

\author{
Autoren \\ Institute \\ 1 Medizinische Klinik I - Pneumologie, Allergologie, \\ Schlaf- und Intensivmedizin, Wuppertaler \\ Lungenzentrum, Petrus-Krankenhaus, Wuppertal \\ 2 Alfried Krupp Krankenhaus Rüttenscheid, \\ Altersmedizin am Alfried Krupp von Bohlen und \\ Halbach Krankenhaus gemeinnützige $\mathrm{GmbH}$, Essen \\ 3 Hermann Buhl Institut für Hypoxie und \\ Schlafmedizinforschung der Universität Innsbruck, \\ Bad Aibling und Eurac Research, Institut für alpine \\ Notfallmedizin, Bozen \\ 4 Fachkrankenhaus Kloster Grafschaft GmbH, \\ Pneumologie II, Akademisches Lehrkrankenhaus \\ der Philipps-Universität Marburg, Schmallenberg \\ 5 Pneumologie, Pneumologische Onkologie, \\ Allergologie, Schlaf- und Beatmungsmedizin, \\ Theresienkrankenhaus, Mannheim
}

S. Stieglitz ${ }^{1}$, H. Frohnhofen ${ }^{2}$, N. Netzer ${ }^{3}$, P. Haidl ${ }^{4}$, M. Orth ${ }^{5}$, A. Schlesinger ${ }^{6}$
6 Klinik für Innere Medizin/ Pneumologie und Beatmungsmedizin, Lungenklinik Köln-Nord, Betriebsteil St. Marien Hospital, Köln

Bibliografie

DOI https://doi.org/10.1055/a-1177-3588 |

Online-Publikation: 20.5.2020 |

Pneumologie 2020; 74: 505-508

(c) Georg Thieme Verlag KG Stuttgart · New York

ISSN 0934-8387

Korrespondenzadresse

Dr. Sven Stieglitz, Chefarzt Medizinische Klinik I -

Pneumologie, Allergologie, Schlaf- und Intensivmedizin,

Wuppertaler Lungenzentrum, Petrus-Krankenhaus

Wuppertal - Akademisches Lehrkrankenhaus

der Heinrich-Heine-Universität Düsseldorf,

Carnaper Str. 48, 42283 Wuppertal

sven.stieglitz@cellitinnen.de
Die epidemiologischen Daten zeigen weltweit, dass von schweren Verläufen und Todesfällen mehrheitlich alte Patienten betroffen sind und die Case Fatality Rate ab einem Alter von 65 Jahren deutlich ansteigt [1]. Die Sterblichkeit bei Patienten über 80 Jahre liegt bei über 15\% [2]. Dies macht COVID-19 zu einer gerontopneumologischen Erkrankung. Die AG pneumologische Altersmedizin gibt im Folgenden daher eine Stellungnahme zur Therapie geriatrischer Patienten mit COVID-19. Diese ist als Ergänzung zu Empfehlungen anderer Arbeitsgruppen zu verstehen.

\section{Klinisches Bild von COVID-19 in der Geriatrie}

Der Schweregrad der Symptomatik reicht auch bei älteren COVID-19 Patienten von milden bis zu sehr schweren Verläufen. Die Latenzzeit bis zum Beginn der klinischen Symptome liegt bei 3-7 Tagen. Trockener Husten und (hohes) Fieber sind die häufigsten Symptome [2]. Milde Diarrhoen und Geschmacksstörungen können auftreten. Diagnostisch auffällig ist eine zuweilen schwere Hypoxämie, die diskordant zu gering ausgeprägten Infiltraten im Röntgenbild oder im CT-Thorax sein kann [3-5]. Diese tritt oft mit einer Latenz von 7 - 10 Tagen nach dem Zeit- punkt der Infektion auf. Pleuraergüsse sind selten durch COVID19 bedingt. Das klinische Bild kann durch Komorbiditäten der geriatrischen Patienten unscharf werden. So kann Dyspnoe bspw. zusätzlich auch durch Herzinsuffizienz oder durch eine chronische Atemwegserkrankung verursacht sein. Daher gilt insbesondere bei geriatrischen Patienten, dass stets überprüft werden muss, ob eine chronische Erkrankung oder die SARSCoV-2-Infektion ursächlich für die Symptome ist. Bei geriatrischen Patienten ist die Schilderung der Symptomatik häufig verschleiert durch kognitive Einschränkungen und durch Sprachschwierigkeiten (z. B. M. Parkinson, Z. n. Apoplex). Immobile Patienten berichteten erst mit erheblicher Latenz von Dyspnoe, da sich Dyspnoe durch das Fehlen körperlicher Belastung erst spät bemerkbar macht [6].

\section{EMPFEHLUNG NR. 1}

Wegen der Komorbiditäten muss ganz besonders bei geriatrischen Patienten differenziert werden, ob die Symptome durch SARS-CoV-2 oder eine andere Erkrankung bedingt sind. 


\section{Medizinisch-ärztliche Versorgung von COVID-19-Patienten}

Die ideale Betreuung geriatrischer COVID-19-Patienten erfolgt primär durch den Pneumologen und erst sekundär durch die Geriatrie. Leider werden nur in wenigen Kliniken beide Disziplinen vorgehalten. In Kliniken, die diese fachliche Ausstattung nicht besetzen, sollte eine einzelne Fachabteilung federführend sein. Die Patienten sollten auch dazu kohortiert werden. Aktuelle Empfehlungen des Robert-Koch-Instituts müssen beachtet werden.

\section{EMPFEHLUNG NR. 2}

Wegen der Dynamik der Erkrankung sollten Patienten mit COVID-19 primär durch einen Pneumologen behandelt werden. Die Weiterverlegung in die Geriatrie und Behandlung durch einen Geriater oder andere Fachabteilungen erfolgt erst sekundär.

\section{Patientenwille und Triage}

Die Berücksichtigung des Patientenwilles und eine Triage von Patienten hinsichtlich invasiver Maßnahmen geschieht in der Geriatrie bereits unabhängig von COVID-19-Routine. Der Patientenwille bezüglich Reanimation und Intubationsbeatmung wird in den meisten geriatrischen Abteilungen bei der stationären Aufnahme regelhaft erfasst und für alle Mitarbeiter sichtbar und schnell erkennbar in der Dokumentation festgehalten. Triage ist auch in der Pneumologie weit verbreitet, wo chronisch Lungenkranke mit „end stage“ Lungenerkrankungen bezüglich Intensivmedizin und Intubation triagiert werden. Sind 2 medizinische Disziplinen in der Patientenversorgung beauftragt, sollte getrennt triagiert werden, um Unterschiede zu erkennen und einen Erfahrungsaustausch zu ermöglichen. Wir empfehlen den Schweregrad der Akuterkrankung nach ASA [7], Karnofsky [8] oder CRB-65-Index zu dokumentieren und bei Patientenaufnahme den chronischen Zustand nach der Frailty Scale [9] zu bewerten. Die Triage bezüglich möglicher therapeutischer Intervention sollte differenziert wie unten aufgeführt erfolgen. Bezüglich etwaiger Patientenverfügungen ist zu prüfen, ob deren dort festgehaltenen Beschlüsse in der aktuellen Situation noch Gültigkeit haben.

\section{EMPFEHLUNG NR. 3}

Die Triage sollte getrennt erfassen, ob folgende Maßnahmen durchgeführten werden sollen:

Intensivtherapie, Intubation/Reanimation, Sauerstoffgabe, High Flow/NIV. Diese therapeutischen Möglichkeiten müssen mit dem Patientenwillen abgeglichen werden. Eventuell auftretende Diskrepanzen sollen umgehend geklärt werden.

\section{Überwachung der Patienten}

Die Patienten der Normalstation brauchen regelmäßig ärztliche und pflegerische Visiten, gerade wenn es sich um Isolierbereiche handelt. Unter Umständen kann auch eine 2-mal tägliche ärztliche Visite notwendig sein, da sich der klinische Status von SARS-CoV-2-Patienten auch rasch verschlechtern kann. Regelmäßige Kontrollen der Sauerstoff-Sättigung per Pulsoxymeter ist sinnvoll (z.B. alle 4 h). Gegebenenfalls können kontinuierlich messende Systeme aus der Schlafmedizin verwendet werden.

\section{Medikamentöse Therapie und Poly- pharmazie}

Kennzeichnend für geriatrische Patienten ist die Polypharmazie. Es ist notwendig, die medikamentöse Therapie hinsichtlich Arzneimittelinteraktion und Nebenwirkungspotenzial zu prüfen. Das gilt umso mehr, wenn überlegt wird, eine medikamentöse Therapie zu beginnen, die keine offizielle Zulassung zur Therapie von SARS-CoV-2 besitzt. Hydroxychloroquin bspw. begünstigt EKG-Veränderungen (long-QT), welche durch Azithromycin aggraviert werden. Das Absetzen von etablierten Medikamenten muss ohne vorliegende Evidenz genauso kritisch gesehen werden (z. B. Absetzen von ACE-Hemmern bei Herzinsuffizienz) $[10,11]$.

Wenn Patienten nur mit einem Vernebler antiobstruktive Medikamente inhalieren können, muss die antiobstruktive Therapie mit Vernebler fortgesetzt werden. Es gibt Untersuchungen, dass die Inhalation von Kochsalz die Viruslast reduziert. Gegebenenfalls kann ein Vernebler verwendet werden, bei dem im Exspirationsschenkel ein Filter aufgesetzt wird. Alternativen sind die Kombination aus Spacer und Dosieraerosol oder der Respimat. Pulver sind bezüglich Umgebungskontamination wahrscheinlich unkritisch.

EMPFEHLUNG NR. 4

Inhalationen mit Vernebler können auch bei Patienten mit COVID-19-Erkrankung eingesetzt werden, wenn dies begleitende Lungenerkrankungen wie COPD erfordern.

\section{Intensivmedizin und respiratorische Therapie}

Die Intensivmedizin hat in den letzten Jahren hinsichtlich der Therapie der ventilatorischen und respiratorischen Insuffizienz einige Fortschritte gemacht. Dazu gehören am weniger invasiven Ende des Spektrums therapeutischer Möglichkeiten die Einführung von High-Flow-Systemen (Applikation von warmer, befeuchteter Luft mit hohem Fluss bei zusätzlicher Gabe von Sauerstoff über eine Nasenkanüle), die nicht-invasive Beatmung NIV und die Bauchlagerung von Patienten sowohl nach Intubation als auch unter NIV oder High Flow. Am anderen Ende des Spektrums steht der teure und invasive Einsatz der extrakorpo- 
ralen Membranoxygenierung ECMO. Für die NIV konnte insbesondere bei aktiven Mund-Nasen-Masken mit zusätzlicher Sauerstoffzufuhr bis $15 \mathrm{l} / \mathrm{min}$ gezeigt werden, dass mit vergleichsweise geringen Kosten die Sterblichkeit unter Vermeidung von intensivmedizinischen Therapiekomplikationen vermindert werden kann. Die NIV hat daher auch einen etablierten Stellenwert gerade in der Geriatrie und der Palliativmedizin [12].

Bei Patienten mit COVID-19 fällt auf, dass das Ausmaß der Hypoxämie oft größer ist, als das radiologische Bild erwarten lassen würde, und die Luftnot geringer ist, als es die Hypoxämie erwarten lassen würde. Es gibt neben der anatomischen (alveoläre Füllung, Mikroembolien [13]) eine funktionelle Komponente. Diskutiert wird, ob die hypoxisch-pulmonale Vasokonstriktion durch die Virusinfektion aufgehoben wird, sodass es zu einer gravierenden Verteilungsstörung kommt [14]. Dies würde erklären, warum so viele geriatrische Patienten so schwer erkranken, da der Ventilations-Perfusions-Mismatch im Alter zunimmt. Überdrucktherapien (CPAP, NIV) hätten hier ihren therapeutischen Ansatz.

Die Empfehlung der DIVI hinsichtlich der respiratorischen Therapie lautet, dass bei fortschreitendem Lungenversagen die Indikation zur HFNC und NIV eher zurückhaltend gestellt wird. Schwere Hypoxämie wird dabei definiert durch einen $\mathrm{PaO}_{2} / \mathrm{FiO}_{2}$-Quotienten von $\leq 200 \mathrm{mmHg}$. Empfohlen wird, die Intubation als sog. Crush Induction durchzuführen. Eine fiberoptische Intubation wird ausdrücklich abgelehnt [15].

Die $\mathrm{FiO}_{2}$ (fraktionierte inspiratorische Sauerstoffkonzentration) ist aber nicht ohne invasive Beatmung zu ermitteln, da die Sauerstoffgabe über Wandanschluss (zentrale Gasleitung), Sauerstoffflaschen oder Konzentrator nur als Fluss (L/min) angegeben werden kann und eine Umrechnung in eine definierte Sauerstofffraktion nicht zuverlässig gelingt. Der Wert lässt auch außer Acht, mit wie viel Ventilation der Sauerstoffpartialdruck erzielt wird. Das kann anhand des $\mathrm{PCO}_{2}$ bestimmt werden: Besteht eine normale Ventilation, liegt der Wert bei 35 $45 \mathrm{mmHg}$. Bei Werten unter $35 \mathrm{mmHg}$ wird eine Mehrventilation erbracht, um den Sauerstoffwert ausreichend hoch zu halten. Somit ist auch das $\mathrm{PCO}_{2}$ ein Indikator für die Schwere der Hypoxämie und sollte daher klinisch berücksichtigt werden.

Therapeutische Empfehlungen sollten nicht alleine anhand von Sättigungswerten getroffen werden, sondern müssen auch die Klinik des Patienten berücksichtigen. So sind insbesondere das Auftreten von (schwerer) Luftnot und Hechelatmung („Rapid shallow breathing“) wichtige Kriterien für therapeutische Entscheidungen [16]. Wahrscheinlich werden die geriatrischen Patienten durch eine konventionelle Intensivtherapie häufiger gefährdet (Sedierung: Delir; Katecholamine: Organischämien) als dass ein Nutzen erbracht wird.

Bei schwerem Atemversagen sind die Atemfrequenz und die Atemtiefe massiv erhöht. Dies übersetzt sich in einen stark negativen Druck (bis $-100 \mathrm{mmHg}$ ). In dieser Situation stützt die Atempumpe den Kreislauf. Das ist klinisch gut daran erkennbar, dass bei Rapid Sequence Intubation [16] in solch einer Situation der Blutdruck sofort massiv abfällt. Nicht selten ist im Anschluss an die Intubation eine Reanimation erforderlich.
Regelmäßig werden daran anschließend hohe Dosen Katecholamine notwendig, um den Kreislauf zu stützen [17,18]. Allesamt eine Situation, die für geriatrische Patienten im Hinblick auf den weiteren Verlauf nachteilig ist. Es ist daher zu diskutieren, ob diese Art der laryngoskopischen Intubation den Patienten nicht schadet im Vergleich bspw. zu einer bronchoskopischen Intubation.

Wir sehen durchaus die Möglichkeit einer Therapie mit NIV und High Flow. NIV sollte bevorzugt mit non-vented NasenMund-Masken (aktive Masken) durchgeführt werden, um Aerosolbildung zu minimieren. Reine Nasenmasken und passive Mund-Nasen-Masken mit offenen Ausatemventilen sind wegen erhöhter Viruskontamination durch den offenen Mund möglichst zu vermeiden. Diese Empfehlung deckt sich mit den -Empfehlungen der europäischen und amerikanischen Fachgesellschaften, die diese Therapieformen in Therapiealgorithmen berücksichtigt haben [19].

\section{EMPFEHLUNG NR. 5}

Geriatrische COVID-19-Patienten mit hypoxämischer Insuffizienz können mit High Flow oder CPAP-behandelt werden. Patienten mit SARS-CoV-2, die hyperkapnisch sind, können mit NIV behandelt werden. Die Kombination aus NIV-/CPAP-Therapie mit Bauchlagerung/überdrehter Seitenlagerung ist eine zusätzliche Option bei schwerer Erkrankung [20]. Intubationskriterien müssen klinische Kriterien sein und dürfen nicht nur über die HämoglobinSauerstoffsättigung definiert werden. Wenn Triage und Wunsch des Patienten es erlauben, kann eine Intubation erfolgen. Die bronchoskopische Intubation kann erwogen werden, wenn ein Kreislaufstillstand durch eine Crush-Intubation zu befürchten ist.

\section{Palliativmedizinische Behandlungskonzepte}

Es ist ärztliche Aufgabe die Indikation zur intensivmedizinischen Behandlung, aber auch für eine Indikation zur Therapiebegrenzung zu stellen. Dies sollte mit fachärztlicher Expertise möglichst im 4-Augen-Prinzip erfolgen. Danach sind die Einwilligungsfähigkeit und der geäußerte Patientenwille zu ermitteln (ggf. mit Unterstützung von Betreuungsbefugten/Vorsorgebevollmächtigten). Im Falle einer klinischen Verschlechterung trotz Erschöpfung der indizierten und gewünschten medizinischen Maßnahmen haben wir die Pflicht, eine bestmögliche Symptomlinderung und ggf. Sterbebegleitung zu gewährleisten. Die symptomorientierte Therapie sollte u.a. Dyspnoe, Angst, Fieber und trockenen Husten im Fokus haben. Auf die gemeinsame Handlungsempfehlung der Deutschen Gesellschaft für Palliativmedizin und der Deutschen Gesellschaft für Pneumologie und Beatmungsmedizin sei verwiesen [21]. Dort wird die symptomatische Therapie von Atemnot, Husten, Rasselatmung, Angst, Unruhe, Agitation und Delir detailliert beschrieben. 
EMPFEHLUNG NR. 6

Bei palliativmedizinischem Ansatz muss eine symptomorientierte Behandlung der Faktoren Dyspnoe, Angst, Fieber und Husten erfolgen.

\section{Versorgung weglaufgefährdeter Patienten}

Ein Problem stellt die Unterbringung COVID-positiver und COVID-verdächtiger weglaufgefährdeter Patienten mit Demenz oder deliranten Symptomen auf den COVID-Isolierstationen in den Akuthäusern oder COVID-Hilfskrankenhäusern dar. Die Situation ist für diese aus ihrer gewohnten Umgebung des stationären Pflegeheims oder des Zuhauses mit ambulanter Pflege herausgerissenen Patienten besonders schwierig, weil die Patienten sich auch ansatzweise nicht mehr orientieren können. Um diese Patienten nicht zu psychiatrisieren oder medikamentös ruhig zu stellen, müssen in Abwägung brandschutzrechtlicher Anforderungen selbstbestimmungsgerechte Maßnahmen getroffen werden, um sie an einer Infektionsübertragung außerhalb der Isolierstationen zu hindern. Auf eine Fixierung am Bett sollte dabei unter allen Umständen verzichtet werden.

\section{EMPFEHLUNG NR. 7}

Weglaufgefährdete geriatrische COVID-19-Patienten sollen auf Isolierstationen nicht fixiert oder medikamentös ruhiggestellt werden. Nach Möglichkeit sind geeignete Maßnahmen zu schaffen, z.B. durch personelle Besetzung an den Notausgangsbereichen bzw. durch Abstimmung mit den Brandschutzbeauftragten, welche Notausgänge unbedingt durchgehend offengehalten werden müssen, um die Patienten am Weglaufen aus dem Isolierbereich in würdiger Form zu hindern.

Interessenkonflikt

Die Autorinnen/Autoren geben an, dass kein Interessenkonflikt besteht.

Literatur

[1] Zhu N, Zhang D, Wang W. China Novel Coronavirus Investigating and Research Team. et al. A Novel Coronavirus from Patients with Pneumonia in China, 2019. N Engl J Med 2020; 382: 727-733. doi:10.1056/NEJMoa2001017

[2] Zhou F, Yu T, Du R et al. Clinical course and risk factors for mortality of adult inpatients with COVID-19 in Wuhan, China: a retrospective cohort study. Lancet 2020; 395: 1054-1062. doi:10.1016/S0140-6736 (20)30566-3

[3] Bernheim A, Mei X, Huang M et al. Chest CT Findings in Coronavirus Disease-19 (COVID-19): Relationship to Duration of Infection. Radiology 2020. doi:10.1148/radiol.2020200463
[4] Chung M, Bernheim A, Mei X et al. CT Imaging Features of 2019 Novel Coronavirus (2019-nCoV). Radiology 2020; 295: 202-207. doi:10.1148/radiol.2020200230

[5] Fang Y, Zhang H, Xie J et al. Sensitivity of Chest CT for COVID-19: Comparison to RT-PCR. Radiology 2020. doi:10.1148/radiol.2020200432

[6] Stieglitz S. Gerontopneumologie: Herausforderungen, Tipps und Tricks. Deutsche Ärzteblatt 2019; 116: [19]. doi:10.3238/PersPneumo.2019.02.15.003

[7] Deiner S, Fleisher LA, Leung JM et al., Anesthesia ASACoG, the ASAPBHIAdherence to recommended practices for perioperative anesthesia care for older adults among US anesthesiologists: results from the ASA Committee on Geriatric Anesthesia-Perioperative Brain Health Initiative ASA member survey. Perioper Med (Lond) 2020; 9: 6. doi:10.1186/s13741-020-0136-9

[8] Zeng A, Song X, Dong J et al. Mortality in Relation to Frailty in Patients Admitted to a Specialized Geriatric Intensive Care Unit. J Gerontol A Biol Sci Med Sci 2015; 70: 1586-1594. doi:10.1093/gerona/glv084

[9] Rockwood K, Song X, MacKnight C et al. A global clinical measure of fitness and frailty in elderly people. CMAJ 2005; 173: 489-495. doi:10.1503/cmaj.050051

[10] Danser AHJ, Epstein M, Batlle D. Renin-Angiotensin System Blockers and the COVID-19 Pandemic: At Present There Is No Evidence to Abandon Renin-Angiotensin System Blockers. Hypertension 2020. doi:10.1161/HYPERTENSIONAHA.120.15082

[11] Shahid Z, Kalayanamitra R, McClafferty B et al. COVID-19 and Older Adults: What We Know. J Am Geriatr Soc 2020. doi:10.1111/ jgs. 16472

[12] Scala R, Ciarleglio G, Maccari U et al. Ventilator Support and Oxygen Therapy in Palliative and End-of-Life Care in the Elderly. Turk Thorac J 2020; 21: 54-60. doi:10.5152/TurkThoracj.2020.201401

[13] Tang N, Bai H, Chen X et al. Anticoagulant treatment is associated with decreased mortality in severe coronavirus disease 2019 patients with coagulopathy. J Thromb Haemost 2020. doi:10.1111/jth.14817

[14] Gattinoni L, Coppola S, Cressoni M et al. Covid-19 Does Not Lead to a “Typical" Acute Respiratory Distress Syndrome. Am J Respir Crit Care Med 2020. doi:10.1164/rccm.202003-0817LE

[15] Kluge $S$, Janssens $U$, Welte $T$ et al. Recommendations for critically ill patients with COVID-19. Med Klin Intensivmed Notfmed 2020; 115 : 175-177. doi:10.1007/s00063-020-00674-3

[16] Pierson DJ. Indications for mechanical ventilation in adults with acute respiratory failure. Respir Care 2002; 47: 249-262; discussion 262245

[17] Skytioti M, Sovik S, Elstad M. Respiratory pump maintains cardiac stroke volume during hypovolemia in young, healthy volunteers. J Appl Physiol (1985) 2018; 124: 1319-1325. doi:10.1152/japplphysiol.01009.2017

[18] Taboada M, Doldan P, Calvo A et al. Comparison of Tracheal Intubation Conditions in Operating Room and Intensive Care Unit: A Prospective, Observational Study. Anesthesiology 2018; 129: 321-328. doi:10.1097/ALN.0000000000002269

[19] COVID-19 Guidelines. SCCM 2020. https://www.sccm.org/SurvivingSepsisCampaign/Guidelines/COVID-19. Accessed: 11.05.2020

[20] Ding L, Wang L, Ma W et al. Efficacy and safety of early prone positioning combined with HFNC or NIV in moderate to severe ARDS: a multi-center prospective cohort study. Crit Care 2020; 24: 28. doi:10.1186/s13054-020-2738-5

[21] Nehls W, Delis S, Haberland B et al. Management of Patients with COVID-19 - Recommendations from a Palliative Care Perspective. Pneumologie 2020. doi:10.1055/a-1156-2759 